\title{
Interesterified palm olein lowers postprandial glucose-dependent insulinotropic polypeptide response in type 2 diabetes
}

\begin{abstract}
Purpose: We aim to investigate the postprandial effects of palm olein (PO) and chemically interesterified palm olein (IPO) with different proportions of palmitic acid at the sn-2 position using high oleic sunflower oil (HOS) as control fat on concentrations of gut hormones, glucose homeostasis, satiety, lipid and inflammatory parameters in type 2 diabetic (T2D) subjects. Methods: Using a randomised double-blind crossover design, $21($ men $=6$, women $=15)$ T2D subjects consumed test meals $(3.65 \mathrm{MJ})$ consisting of a high fat muffin (containing $50 \mathrm{~g}$ test fats provided as PO, IPO or HOS) and a milkshake. Postprandial changes in gut hormones, glucose homeostasis, satiety, lipid and inflammatory parameters after meals were analysed. Some of the solid fractions of the IPO were removed and thus the fatty acid composition of the PO and IPO was not entirely equal (PO vs IPO: palmitate 39.8 vs 38.7; oleate 43.6 vs 45.1). PO, IPO and HOS contained 9.7, 38.9 and $0.2 \mathrm{~g} / 100 \mathrm{~g}$ total fatty acids of palmitic acid at the sn-2 position, respectively. At $37{ }^{\circ} \mathrm{C}$, IPO contained $4.2 \%$ SFC whereas PO and HOS were completely melted. Results: Our novel observation shows that the incremental area under curve (iAUC) 0-6 h of plasma GIP concentration was on average 16\% lower following IPO meal compared with PO and HOS (P < 0.05) meals. Serum C-peptide concentrations exhibited a significant meal $\times$ gender interaction $(\mathrm{P}=0.009)$. No differences between test meals were noted for other measurements. Conclusions: This study shows no adverse effect of interesterification on hormones associated with glucose homeostasis notably GLP-1 in T2D subjects.
\end{abstract}

Keyword: Glucose-dependent insulinotropic polypeptide; Gut hormones; Glucose metabolism; Interesterified palm olein; Type 2 diabetes 